\title{
The subword complexity of a two-parameter family of sequences
}

\author{
Aviezri S. Fraenkel, Tamar Seeman \\ Department of Computer Science and Applied Mathematics \\ The Weizmann Institute of Science \\ Rehovot 76100, Israel \\ fraenkel, tamars@wisdom.weizmann.ac.il \\ http://www.wisdom.weizmann.ac.il/〜fraenkel, 〜tamars \\ Jamie Simpson \\ School of Mathematics, Curtin University \\ Perth WA 6001, Australia \\ simpson@cs. curtin. edu . au \\ http://www.cs.curtin.edu.au/〜 simpson
}

RECEIVED: $4 / 14 / 2000$

ACCEPTED: 2/06/2001

\begin{abstract}
We determine the subword complexity of the characteristic functions of a twoparameter family $\left\{A_{n}\right\}_{n=1}^{\infty}$ of infinite sequences which are associated with the winning strategies for a family of 2-player games. A special case of the family has the form $A_{n}=\lfloor n \alpha\rfloor$ for all $n \in \mathbb{Z}_{>0}$, where $\alpha$ is a fixed positive irrational number. The characteristic functions of such sequences have been shown to have subword complexity $n+1$. We show that every sequence in the extended family has subword complexity $O(n)$.
\end{abstract}

\section{Introduction}

Denote by $\mathbb{Z}_{>0}$ and $\mathbb{Z}_{>0}$ the set of nonnegative integers and positive integers respectively. Given two heaps of finitely many tokens, we define a 2-player heap game as follows. There are two types of moves: 
1. Remove any positive number of tokens from a single heap.

2. Remove $k>0$ tokens from one heap and $l>0$ from the other. Here $k$ and $l$ are constrained by the condition: $0<k \leq l<s k+t$, where $s$ and $t$ are predetermined positive integers.

The player who reaches a state where both heaps are empty wins. The special case $s=t=1$ is the classical Wythoff game [15], [16], [5].

Fraenkel showed [11] that every possible position in a game of this type can be classified as either a $P$-position, in which the Previous player can win, or an $N$-position, in which the Next player can win. Thus a winning strategy involves moving from an $N$-position to a $P$-position. Let $\mathcal{P}$ denote the set of all possible $P$-positions in a game with given values for $s$ and $t$. Let mex $S$ denote the least nonnegative integer in $\mathbb{Z}_{\geq 0} \backslash S$.

Then $\mathcal{P}=\bigcup_{i=0}^{\infty}\left\{\left(A_{i}, B_{i}\right)\right\}$, where for every $n \in \mathbb{Z}_{\geq 0}$,

$$
A_{n}=\operatorname{mex}\left\{\left\{A_{i}: 0 \leq i<n\right\} \cup\left\{B_{i}: 0 \leq i<n\right\}\right\}, \quad B_{n}=s A_{n}+t n .
$$

Thus $A_{n}$ and $B_{n}$ are strictly increasing sequences, with $A_{0}=B_{0}=0$ and $A_{1}=1$ for all $s, t \in \mathbb{Z}_{>0}$. Denoting $A=\bigcup_{i=1}^{\infty} A_{i}$ and $B=\bigcup_{i=1}^{\infty} B_{i}$, we have $A \cup B=\mathbb{Z}_{>0}$, and $A \cap B=\emptyset$.

Fraenkel [9] generalized the classical Wythoff game $(s=t=1)$ to the case $s=1, t \geq$ 1 , and showed that a polynomial-time-computable strategy exists for the game. The strategy is based on the Ostrowski numeration system [12], with a base computed from the simple continued fraction expansion of $\alpha$, where $\alpha$ satisfies $A_{n}=\lfloor n \alpha\rfloor$ for all $n \geq 0$. Fraenkel showed [11] that such $\alpha$ exists if and only if $s=1$, but that a polynomial-timecomputable strategy based on a numeration system defined by certain recursion formulas [10] nevertheless exists for every $s, t \in \mathbb{Z}_{>0}$.

In this paper we investigate an additional property of the class of heap games for general $s, t \in \mathbb{Z}_{>0}$ : the subword complexity of the characteristic function of $A$. For fixed $s, t \in \mathbb{Z}_{>0}$, define the characteristic function of $A$ as $\chi=\chi(A): \mathbb{Z}_{>0} \longrightarrow\{0,1\}$, where

$$
\chi(x)= \begin{cases}1, & x \in A \\ 0, & x \notin A .\end{cases}
$$

A word $w$ is a factor of $y$ if there exist words $u, v$, possibly empty, such that $y=u w v$. Define the subword complexity function $c_{s, t}: \mathbb{Z}_{>0} \longrightarrow \mathbb{Z}_{>0}$, where $c=c_{s, t}(n)=$ number of distinct factors of length $n$ of the infinite sequence $\chi$. Our goal is to determine the subword complexity function $c$ of $\chi$ for general $s, t \in \mathbb{Z}_{>0}$. 
The problem of computing the subword complexity of a given sequence has been addressed in a number of earlier works. For a survey of results in this area, we refer the reader to [2] and [8]. In particular, [13] contains an analysis of the subword complexity of infinite sequences $S$ of the form $S=f^{\omega}(b)$, where $f$ is a morphism such that $b \in\{0,1\}$ is a prefix of $f(b)$. For example, it is shown there that if the functions

$$
f_{0}(n)=\left|f^{n}(0)\right|, \quad f_{1}(n)=\left|f^{n}(1)\right|
$$

have asymptotic growth rate $\Theta\left(k^{n}\right)$ for some constant $k$, then $S$ has linear subword complexity.

In section 2 we show that for every $s, t \in \mathbb{Z}_{>0}, \chi$ is generated by such a morphism $f$. In section 4 it is shown that both $\left|f^{n}(0)\right|$ and $\left|f^{n}(1)\right|$ have asymptotic growth rate $\Theta\left(k^{n}\right)$. Thus by [13], $\chi$ has linear subword complexity for all $s, t \in \mathbb{Z}_{>0}$. This is consistent with our result that for all $s, t \in \mathbb{Z}_{>0}, c(n+1)-c(n) \in\{1,2\}$ for every $n \in \mathbb{Z}_{>0}$.

For every given $s, t \in \mathbb{Z}_{>0}$, the set of positive integers consists of intervals over which $c(n+1)-c(n)=2$ for all $n$, alternating with intervals over which $c(n+1)-c(n)=1$ for all $n$. In other words, there exist intervals of "fast growth" of $c(n)$ relative to $n$, alternating with intervals of relatively "slow growth". By computing $c(n)$ at the first point of every interval of fast growth, we found that the subword complexity at these points converges asymptotically to

$$
E\left(\left(1-\frac{s-1}{(s+t-1) \alpha}\right)+\left(1+\frac{s-1}{(s+t-1) \alpha}\right) n\right)
$$

where $E(x)$ denotes the closest integer to $x$, and $\alpha>1$ is a constant defined below in (2). Similarly, the complexity at the first point of every interval of slow growth converges to

$$
E\left(\left(1+\frac{(s-1) \alpha}{(2 s+t-2) \alpha-(s-1)}\right) n+1\right) .
$$

These two limits are respectively the lower and upper bounds on the asymptotic subword complexity of $\chi$. When $s=1$, the intervals of fast growth of $c(n)$ are empty, so the lower and upper bounds are equivalent and we have $c(n)=n+1$ for all $n \in \mathbb{Z}_{>0}$.

In section 3 we introduce the concept of special words, and show how to determine the number of distinct special factors of $\chi$ of any given length. In section 4 we use the results of section 3 to determine the subword complexity of $\chi$. The subword complexity formula is presented both for finite $n$, and as an asymptotic value when $n$ approaches $\infty$.

\section{Preliminaries}

In this section, we describe a morphism, $f$, which generates $\chi$ for fixed $s$ and $t$. 


\subsection{An Equivalent Sequence}

A morphism $h$ is called non-erasing if $h(u) \geq 1$ for every word $u$. See e.g. [14].

Definition. For given values of $s$ and $t$, let $f:\{0,1\}^{*} \longrightarrow\{0,1\}^{*}$ be a morphism defined by the following rules:

(i) $f(0)=1^{s}$ (concatenation of 1 by itself $s$ times),

(ii) $f(1)=1^{s+t-1} 0$.

Note that the morphism thus defined is non-erasing. Further, $f(u \cdot v)=f(u) \cdot f(v)$, where "." (usually omitted), denotes concatenation. We use standard function iteration: $f^{0}(u)=u$, and $f^{i}(u)=f\left(f^{i-1}(u)\right)$ for $i \in \mathbb{Z}_{>0}$. So also $h^{i}(u \cdot v)=h^{i}(u) \cdot h^{i}(v)$ for all $i \in \mathbb{Z}_{\geq 0}$.

Notation. Let $\epsilon$ denote the empty word. Then $1^{0}=0^{0}=\epsilon$, and for all $i \in \mathbb{Z}_{\geq 0}$, $f^{i}(\epsilon)=\epsilon$.

Since $f$ is a non-erasing morphism and $f(1)=1 x \quad\left(x=1^{s+t-2} 0\right)$, we can define $F=$ $f^{\omega}(1)=1 x f(x) f^{2}(x) f^{3}(x) \cdots$, the unique infinite string of which $f(1), f^{2}(1), f^{3}(1), \ldots$ are all prefixes [6].

Theorem 1. $F=\chi(A)$.

To prove our theorem, we apply the following result.

Lemma 1. Suppose that for some $n \in \mathbb{Z}_{>0}$, the $n$-th one in $F$ is at position $k$. Then the $n$-th zero is at position $s k+t n$.

Proof. If the $n$-th one is at position $k$, then the length $k$ prefix contains $n$ ones and $k-n$ zeros. Since $F=f^{\omega}(1)$, we can apply the morphism to this prefix to obtain another (longer) prefix of $F$. This prefix will contain $n$ copies of $f(1)$ and $k-n$ of $f(0)$, and so has $n$ zeros, $n(s+t-1)+(k-n) s$ ones, and length $n+n(s+t-1)+(k-n) s=n t+k s$. Since it ends with $f(1)$ which ends in zero, the $n$-th zero is in position $n t+k s$.

Proof of Theorem 1. We show by induction that for all $n \in \mathbb{Z}_{>0}$, the $n$-th one is at position $A_{n}$, and the $n$-th zero is at position $B_{n}$ in $F$. 
(i) $n=1: A_{1}=1$, and the first one is at position 1 . Thus by Lemma 1 , the first zero is at position $s+t=B_{1}$.

(ii) $n>1$ : Suppose that for all $i<n$, the $i$-th one and the $i$-th zero are at positions $A_{i}$ and $B_{i}$ respectively. From the definition of the $A_{i}$ sequence, $A_{n}$ is the least integer distinct from $A_{i}$ and $B_{i}$ for all $i<n$; thus either the $n$-th zero or the $n$-th one occurs at bit position $A_{n}$, with the other bit occurring at some later position. But Lemma 1 implies that the $n$-th one occurs earlier than the $n$-th zero, so it must be in position $A_{n}$, and by the definition of $B_{n}$, the $n$-th zero is in position $B_{n}$.

Thus for every $x \in \mathbb{Z}_{>0}$, the bit at position $x$ of $F$ is a 1 if and only if $\chi(x)=1$, where $\chi$ is the characteristic sequence of $A$.

\subsection{Properties of $F$}

For the remainder of this paper, we determine the subword complexity of $\chi$ by analyzing $F$. To do so, we first collect several properties of $F$ which are implied by the rules of the generating morphism $f$.

Lemma 2. F consists of isolated 0 -bits separated by $1^{s+t-1}$ or by $1^{2 s+t-1}$.

Proof. The only way to generate a 0 is as the termination of $f(1)=1^{s+t-1} 0$. Thus every 0 is preceded by $1^{s+t-1}$, and is followed by either $f(1)$ or $f(0)$, so 00 is not a factor of $F$. Therefore every 0 is followed by either $f(1)$ or $f(0) f(1)$. If it is followed by $f(1)$, then it is separated from the next 0 -bit by $1^{s+t-1}$. If it is followed by $f(0) f(1)$, then it is separated from the next 0 -bit by $1^{2 s+t-1}$.

Lemma 3. If $f(x)=f(y)$ then $x=y$.

Proof. Let $x=x_{1} x_{2} \cdots x_{m}$ and $y=y_{1} y_{2} \cdots y_{n}$ and suppose $f(x)=f(y)$. Then we have

$$
f\left(x_{1}\right) f\left(x_{2}\right) \cdots f\left(x_{m}\right)=f\left(y_{1}\right) f\left(y_{2}\right) \cdots f\left(y_{n}\right) .
$$

If $f\left(x_{m}\right)$ ends in a zero, then it must be $f(1)$ and so $x_{m}=1$. Otherwise $x_{m}=0$. The same applies to $y_{n}$ and so we must have $x_{m}=y_{n}$ and

$$
f\left(x_{1}\right) f\left(x_{2}\right) \cdots f\left(x_{m-1}\right)=f\left(y_{1}\right) f\left(y_{2}\right) \cdots f\left(y_{n-1}\right) .
$$

Continuing inductively we get $x_{m-1}=y_{n-1}$ and so on, giving $x=y$. 
Remark. If $x=f(w)$, then by Lemma $3 w$ is the unique inverse of $x$, which we denote $f^{-1}(x)$.

Notation. Denote by $|w|$ the length of the factor $w$, i.e., the number of its letters, counting multiplicities.

Lemma 4. Let $w$ be a factor of $F$ beginning with $f(0) f(1)$ or $f(1)$, and terminating with $f(1)$. Then $f^{-1}(w)$ exists, and $\left|f^{-1}(w)\right|<|w|$.

Proof. Suppose that the assertion holds for all $w$ terminating with $f(1)$, with $|w| \leq n$. Let $w$ be any factor of length $n+1(n \geq|f(1)|)$, beginning with $f(0) f(1)$ or $f(1)$ and terminating with $f(1)$. We consider two cases.

(i) $w$ begins with $f(1)$. Then $w=f(1) w^{\prime} f(1)$. By Lemma 2, if $w^{\prime}$ is nonempty, then $w^{\prime}$ begins with $f(0) f(1)$ or $f(1)$, and $\left|w^{\prime} f(1)\right|=n-s-t+1<n$. Then by the induction hypothesis, $f^{-1}(w)=1 f^{-1}\left(w^{\prime} f(1)\right)$, and $\left|1 f^{-1}\left(w^{\prime} f(1)\right)\right|<\left|f(1) w^{\prime} f(1)\right|$.

(ii) $w$ begins with $f(0) f(1)$, so $w=f(0) f(1) w^{\prime} f(1)$. The argument is as in the case (i) with an extra prefix $f(0)$.

\section{Special Words}

As stated in the introduction, our goal is to determine the subword complexity function $c$ of $F$. Recall that for every $n \in \mathbb{Z}_{>0}, c(n)$ denotes the number of distinct words of length $n$ which are factors of $F$. Thus $c(1)=2$, and for every $n \in \mathbb{Z}_{>0}, c(n+1)-c(n)$ is the number of length $n$ factors of $F$ that can be followed by both a 0 and a 1 in $F$.

Definition. Following standard terminology, define a factor $w$ of $F$ to be special if both $w 0$ and $w 1$ are factors of $F$. If $w$ can be extended only by adjoining one of 0,1 , then $w$ is nonspecial.

Remark. $x$ is special $\Longleftrightarrow$ every suffix of $x$ is special.

Definition. Let $N: \mathbb{Z}_{>0} \longrightarrow \mathbb{Z}_{\geq 0}$ be the function defined as follows. For every $n \in \mathbb{Z}_{>0}$, $N(n)$ denotes the number of distinct special words of length $n$. 
Thus for all $n \in \mathbb{Z}_{>0}, c(n+1)-c(n)=N(n)$, so

$$
c(n)=c(1)+\sum_{i=1}^{n-1}(c(i+1)-c(i))=2+\sum_{i=1}^{n-1} N(i) .
$$

To determine the subword complexity of $F$, therefore, we first compute $N(n)$ for every $n \in \mathbb{Z}_{>0}$.

Notation. Let $x_{0}$ denote $1^{s+t-1}$, and $x_{1}$ denote $1^{2 s+t-2}$.

Note that $s=1 \Longrightarrow x_{0}=x_{1}=1^{t}$.

Remark. If $k<2 s+t-1$ then $1^{k}$ is special. In particular, $x_{0}$ and $x_{1}$ are special.

Definition. Given $x, y \in F, x$ possibly nonempty. Then $x$ is said to be a proper prefix of $y$ if $y=x u$ for some nonempty $u$. Similarly, $x$ is a proper suffix of $y$ if $y=u x$ for some nonempty $u$.

Lemma 5. Given any word $w=b u \in F, b \in\{0,1\}$. Suppose that $u$ is special and $w$ is nonspecial. Then either $u=1^{k}$ for some $k<2 s+t-1$, or $f(1)$ is a proper prefix of $u$.

Proof. Since $u$ is extendible in two possible ways, whereas bu is extendible in only one way, it follows that both $0 u$ and $1 u$ are factors of $F$. Suppose that $u$ contains no 0 . Then Lemma 2 implies that $|1 u|<2 s+t$, so we have $u=1^{k}, k<2 s+t-1$. On the other hand, suppose that $u$ contains at least one 0 . Then $u$ begins with $1^{m} 0$ for some $m \in \mathbb{Z}_{\geq 0}$. Since $0 u \in F$, Lemma 2 implies that $m \in\{2 s+t-1, s+t-1\}$. But since $1 u \in F$, Lemma 2 implies that $m<2 s+t-1$. Thus $m=s+t-1$ and $u$ begins with $1^{s+t-1} 0=f(1)$.

Definition. For given $s$ and $t$, define $g:\{0,1\}^{*} \longrightarrow\{0,1\}^{*}$, where for all $x \in\{0,1\}^{*}$, $g(x)=f(x) 1^{s+t-1}$.

Lemma 6. $x$ special $\Longleftrightarrow g(x)$ special. 


\section{Proof.}

(i) Suppose that $x$ is special, so both $x 0$ and $x 1$ are factors of $F$. Then Lemma 2 implies that both $x 01$ and $x 1$, and thus $f(x) f(01)$ and $f(x) f(1)$, are factors of $F$. But

$$
f(x) f(01)=f(x) 1^{s} 1^{s+t-1} 0=f(x) 1^{s+t-1} 1^{s} 0=g(x) 1^{s} 0,
$$

and

$$
f(x) f(1)=f(x) 1^{s+t-1} 0=g(x) 0 .
$$

Therefore both $g(x) 1$ and $g(x) 0$ are factors of $F$, so $g(x)$ is special.

(ii) Suppose that $g(x)=f(x) 1^{s+t-1}$ is special. Then both $g(x) 0$ and $g(x) 1$ are factors of $F$. But

$$
g(x) 0=f(x) 1^{s+t-1} 0=f(x) f(1),
$$

so by Lemma $4, f^{-1}(f(x) f(1))=x 1$ is a factor of $F$.

Suppose that $x=x^{\prime} 0$, for some $x^{\prime}$. Then $g(x) 1=f\left(x^{\prime}\right) f(0) 1^{s+t}=f\left(x^{\prime}\right) 1^{2 s+t}$, contradicting Lemma 2. Thus $x=x^{\prime} 1$ for some $x^{\prime}$, so

$$
g(x) 1=f\left(x^{\prime}\right) f(1) 1^{s+t}=f\left(x^{\prime}\right) 1^{s+t-1} 01^{s+t} .
$$

Since $s+t-1<s+t$, Lemma 2 implies that the $01^{s+t}$ terminating $g(x) 1$ is followed by $1^{s-1} 0$, to form

$$
f\left(x^{\prime}\right) 1^{s+t-1} 01^{2 s+t-1} 0=f(x 01) .
$$

Thus $f^{-1}(f(x 01))=x 01$ is a factor of $F$, so $x$ is special.

Corollary 1. $x$ is special $\Longleftrightarrow$ for all $i \in \mathbb{Z}_{\geq 0}$, every suffix of $g^{i}(x)$ is special.

Proof. obtain:

$x$ special $\Longleftrightarrow g(x)$ special $\Longleftrightarrow g^{2}(x)$ special $\Longleftrightarrow \cdots \Longleftrightarrow g^{i}(x)$ special,

for all $i \in \mathbb{Z}_{\geq 0}$. But a word is special if and only if all of its suffixes are special, so our result follows.

Theorem 2. $w$ is special $\Longleftrightarrow w$ is a suffix of $g^{i}\left(x_{1}\right)$ for some $i$.

Proof. $i \in \mathbb{Z}_{\geq 0}$, every suffix of $g^{i}\left(x_{1}\right)$ is special.

In the other direction, suppose that $w$ is special. If $w$ contains no zeros, then by Lemma 2, $w$ must have the form $1^{k}$ for some $k \leq 2 s+t-2$, so $w$ is a suffix of $x_{1}=g^{0}\left(x_{1}\right)$. 
We prove the other cases by induction on $|w|$, the start of the induction being the case above. Suppose $w$ contains a zero and both $w 0$ and $w 1$ occur in $F$. By Lemma $2, w$ must end in $1^{s+t-1}$, so let

$$
w=w[1] w[2] \cdots w[k] 1^{s+t-1},
$$

where $w[j]$ denotes the $j$-th bit of $w$. Again by Lemma 2 we see that $w[k]=0$ and therefore $w[k-s-t+1] \cdots w[k]=f(1)$. We then consider $w[k-s-t]$. If this is zero then $w[1] \cdots w[k-s-t]$ ends in $f(1)$ or a suffix of $f(1)$; otherwise it ends in $f(0)$ or a suffix of $f(0)$. Going backwards in this way we can uniquely identify $w$ as having the form

$$
w=v f\left(u_{1}\right) f\left(u_{2}\right) \cdots f\left(u_{m}\right) 1^{s+t-1},
$$

where $v$ is a nonempty suffix of $f(0)$ or $f(1)$. Say it is a suffix of $f\left(u_{0}\right)$, and denote by $x v f\left(u_{1}\right) \cdots f\left(u_{m}\right) 1^{s+t-1}$ the longest special suffix of $f\left(u_{0}\right) f\left(u_{1}\right) \cdots f\left(u_{m}\right) 1^{s+t-1}$. If $|x v|<$ $\left|f\left(u_{0}\right)\right|$, then by Lemma $5, x v f\left(u_{1}\right) \cdots f\left(u_{m}\right) 1^{s+t-1}$ begins with $f(1)$. But this contradicts the fact that $x v$ is a proper suffix of $f\left(u_{0}\right)$.

Thus $f\left(u_{0}\right) f\left(u_{1}\right) \cdots f\left(u_{m}\right) 1^{s+t-1}=g\left(u_{0} \cdots u_{m}\right)$ is special, so by Lemma $6, u_{0} \cdots u_{m}$ is special. Therefore by the induction hypothesis, $u_{0} \cdots u_{m}$ is a suffix of $g^{i}\left(x_{1}\right)$ for some $i$. Since $w$ is a suffix of $f\left(u_{0}\right) f\left(u_{1}\right) \cdots f\left(u_{m}\right) 1^{s+t-1}=g\left(u_{0} \cdots u_{m}\right)$, this implies that $w$ is a suffix of $g^{i+1}\left(x_{1}\right)$.

For every $n \in \mathbb{Z}_{>0}$, define the set

$$
S_{n}=\left\{w:|w|=n \text {, and for some } i \in \mathbb{Z}_{\geq 0}, w \text { is a suffix of } g^{i}\left(x_{1}\right)\right\} .
$$

Theorem 2 implies that $N(n)=\left|S_{n}\right|$ for all $n \in \mathbb{Z}_{>0}$.

\section{Theorem 3.}

$$
N(n)= \begin{cases}2 & \text { if for some } i \in \mathbb{Z}_{\geq 0},\left|g^{i}\left(x_{0}\right)\right|<n \leq\left|g^{i}\left(x_{1}\right)\right|, \\ 1 & \text { otherwise. }\end{cases}
$$

To prove the theorem, we apply several results.

Lemma 7. (a) For all $i \in \mathbb{Z}_{>0}$ and $w, g^{i}(w)=f^{i}(w) g^{i-1}\left(x_{0}\right)$.

(b) For all $i \in \mathbb{Z}_{>0}$ and $w, g^{i}(w)=f^{i}(w) f^{i-1}\left(x_{0}\right) \cdots f^{1}\left(x_{0}\right) f^{0}\left(x_{0}\right)$.

(c) For all $i \in \mathbb{Z}_{\geq 0}$ and $x, y, g^{i}(x y)=f^{i}(x) g^{i}(y)$.

Proof. By induction on $i$.

Corollary 2. (a) Let $i, j \in \mathbb{Z}_{\geq 0}$, with $i \leq j$. Then $g^{i}\left(x_{0}\right)$ is a suffix of $g^{j}\left(x_{0}\right)$. 
(b) Let $k, m \in \mathbb{Z}_{>0}$, with $k \leq m$. Then for all $i \in \mathbb{Z}_{\geq 0}, g^{i}\left(1^{k}\right)$ is a suffix of $g^{i}\left(1^{m}\right)$.

(c) For all $i \in \mathbb{Z}_{\geq 0},\left|g^{i}\left(x_{0}\right)\right| \leq\left|g^{i}\left(x_{1}\right)\right|<\left|g^{i+1}\left(x_{0}\right)\right|$, with $\left|g^{i}\left(x_{0}\right)\right|=\left|g^{i}\left(x_{1}\right)\right|$ if and only if $s=1$.

\section{Proof.}

(a) By Lemma 7(b), $g^{j}\left(x_{0}\right)=f^{j}\left(x_{0}\right) \cdots f^{i+1}\left(x_{0}\right) g^{i}\left(x_{0}\right)$.

(b) Let $\ell=m-k$. Lemma 7(c) implies that

$$
g^{i}\left(1^{m}\right)=g^{i}\left(1^{l} 1^{k}\right)=f^{i}\left(1^{l}\right) g^{i}\left(1^{k}\right) .
$$

(c) By Lemma 7(c), $g^{i}\left(x_{1}\right)=g^{i}\left(1^{s-1} x_{0}\right)=f^{i}\left(1^{s-1}\right) g^{i}\left(x_{0}\right)$. Thus $\left|g^{i}\left(x_{0}\right)\right| \leq\left|g^{i}\left(x_{1}\right)\right|$, with equality if and only if $s=1$. Lemma 7 (a) implies that $g^{i+1}\left(x_{0}\right)=f^{i+1}\left(x_{0}\right) g^{i}\left(x_{0}\right)$, so to prove that $\left|g^{i}\left(x_{1}\right)\right|<\left|g^{i+1}\left(x_{0}\right)\right|$, it suffices to show that $\left|f^{i}\left(1^{s-1}\right)\right|<\left|f^{i+1}\left(x_{0}\right)\right|$. But this is satisfied, since $\left|f^{i}\left(1^{s-1}\right)\right|<\left|f^{i}\left(x_{0}\right)\right|<\left|f^{i+1}\left(x_{0}\right)\right|$.

Notation. By $x \in F$, we mean that $x$ is a factor of $F$.

Proof of Theorem 3. Suppose that $n \leq s+t-1=\left|g^{0}\left(x_{0}\right)\right|$. We show that $\left|S_{n}\right|=1$. Now, $g^{0}\left(x_{1}\right)=x_{1}$ terminates with $x_{0}$, and Lemma $7(\mathrm{~b})$ implies that for all $i \in \mathbb{Z}_{>0}, g^{i}\left(x_{1}\right)$ terminates with $x_{0}$, which terminates with $1^{|n|}$. Thus $1^{|n|}$ is the unique member of $S_{n}$, so $\left|S_{n}\right|=1$.

Let $i \in \mathbb{Z}_{\geq 0}$. Consider the set of $n$ satisfying

$$
\left|g^{i}\left(x_{0}\right)\right|<n \leq\left|g^{i+1}\left(x_{0}\right)\right| .
$$

Then by Corollary 2(a), for all $n$ in the set, $n>\left|g^{0}\left(x_{0}\right)\right|=s+t-1$.

Given some $n$ in the set, denote by $w$ the suffix of length $n$ of $g^{i+1}\left(x_{0}\right)$. Corollary 2(a) implies that $w$ is a suffix of $g^{j}\left(x_{0}\right)$ for all $j \geq i+1$. Thus by Corollary 2(b), $w$ is a suffix of $g^{j}\left(x_{1}\right)$ for all $j \geq i+1$, so we have $w \in S_{n}$.

If there exists a second member of $S_{n}$ distinct from $w$, then this member of $S_{n}$ is a suffix of $g^{j}\left(x_{1}\right)$ for some $j \leq i$. Now, Corollary 2(c) implies that for every positive integer $j<i$,

$$
\left|g^{j}\left(x_{0}\right)\right| \leq\left|g^{j}\left(x_{1}\right)\right|<\left|g^{j+1}\left(x_{0}\right)\right| \leq \cdots \leq\left|g^{i}\left(x_{0}\right)\right| .
$$

Since $n>\left|g^{i}\left(x_{0}\right)\right|$, it follows that if there exists a member of $S_{n}$ distinct from $w$, then this member is a suffix of $g^{i}\left(x_{1}\right)$. Thus $\left|S_{n}\right| \in[1,2]$.

Now, by Corollary 2(c), we have $\left|g^{i}\left(x_{0}\right)\right| \leq\left|g^{i}\left(x_{1}\right)\right|<\left|g^{i+1}\left(x_{0}\right)\right|$. It follows that either (i) $\left|g^{i}\left(x_{0}\right)\right|<n \leq\left|g^{i}\left(x_{1}\right)\right|$, or (ii) $\left|g^{i}\left(x_{1}\right)\right|<n \leq\left|g^{i+1}\left(x_{0}\right)\right|$. Since $N(n)=\left|S_{n}\right|$ for all $n$, it suffices to show that $\left|S_{n}\right|=2$ in case (i), and $\left|S_{n}\right|=1$ in case (ii). 
(i) Suppose that $\left|g^{i}\left(x_{0}\right)\right|<n \leq\left|g^{i}\left(x_{1}\right)\right|$. We assume that $s \geq 2$, because otherwise the set of $n$ satisfying this inequality is empty. Denote by $w^{\prime}$ the length- $n$ suffix of $g^{i}\left(x_{1}\right)$. To prove that $\left|S_{n}\right|=2$, we show that $w$ and $w^{\prime}$ are distinct.

Let $z=g^{i}\left(x_{0}\right)$. Then by Lemma $7(\mathrm{a})$ and (c), we have

$$
\begin{gathered}
g^{i}\left(x_{1}\right)=g^{i}\left(1^{s-1} x_{0}\right)=f^{i}\left(1^{s-1}\right) z, \\
g^{i+1}\left(x_{0}\right)=f^{i+1}\left(x_{0}\right) z .
\end{gathered}
$$

Thus $w$ is a suffix of $f^{i+1}\left(x_{0}\right) z$, and $w^{\prime}$ is a suffix of $f^{i}\left(1^{s-1}\right) z$. Moreover, $n>|z|$, so to prove that $w$ and $w^{\prime}$ are distinct, it is sufficient to show that the rightmost bits of $f^{i}\left(1^{s-1}\right)$ and $f^{i+1}\left(x_{0}\right)$ differ.

For every $x \in F$, if $x$ terminates with 1 , then $f(x)$ terminates with $f(1)=1^{s+t-1} 0$, which ends in 0. Similarly, if $x$ terminates with 0 , then $f(x)$ terminates with $f(0)=$ $1^{s}$, which ends in 1 . Thus for all $i \in \mathbb{Z}_{\geq 0}$, the rightmost bits of $f^{i}(1)$ and $f\left(f^{i}(1)\right)=$ $f^{i+1}(1)$ differ. But since $f$ is a morphism, $f^{i+1}\left(x_{0}\right)$ terminates with $f^{i+1}(1)$, and $f^{i}\left(1^{s-1}\right)$ terminates with $f^{i}(1)$. Thus the rightmost bit of $f^{i}\left(1^{s-1}\right)$ differs from that of $f^{i+1}\left(x_{0}\right)$. It follows that $w$ and $w^{\prime}$ are distinct, so for all $n$ satisfying $\left|g^{i}\left(x_{0}\right)\right|<$ $n \leq\left|g^{i}\left(x_{1}\right)\right|,\left|S_{n}\right|=2$.

(ii) Suppose that $\left|g^{i}\left(x_{1}\right)\right|<n \leq\left|g^{i+1}\left(x_{0}\right)\right|$. Since $n>\left|g^{i}\left(x_{1}\right)\right|$, there does not exist a suffix of length $n$ of $g^{i}\left(x_{1}\right)$. Thus $w$ is the only member of $S_{n}$, so $\left|S_{n}\right|=1$.

Example. Let $s=2, t=1$. Then $f(1)=110, f(0)=11, x_{0}=11$ and $x_{1}=111$. Thus

(i) $g\left(x_{0}\right)=11011011$, so $\left|g\left(x_{0}\right)\right|=8$,

(ii) $g\left(x_{1}\right)=11011011011$, so $\left|g\left(x_{1}\right)\right|=11$,

(iii) $g^{2}\left(x_{0}\right)=110110111101101111011011$, so $\left|g^{2}\left(x_{0}\right)\right|=24$.

Note that both $g\left(x_{1}\right)$ and $g^{2}\left(x_{0}\right)$ terminate with $g\left(x_{0}\right)$ - consistent with parts (a) and (b) of Corollary 2.

Now, for example, $\left|g\left(x_{0}\right)\right|<10 \leq\left|g\left(x_{1}\right)\right|$, so we show that $\left|S_{10}\right|=2$. The suffix of length 10 of $g^{2}\left(x_{0}\right)$ is 1111011011, which we denote $w$. In fact, Corollary 2 implies that $g^{2}\left(x_{0}\right)$, and thus $w$, is a suffix of $g^{j}\left(x_{1}\right)$ for all $j \geq 2$. Thus the suffix $w^{\prime}=1011011011$ of $g\left(x_{1}\right)$ is the only member of $S_{10}$ which is distinct from $w$. Both $w$ and $w^{\prime}$ terminate with $g\left(x_{0}\right)=11011011$, but they differ in the bit immediately preceding $g\left(x_{0}\right)$, so they are distinct. Thus $\left|S_{10}\right|=2$.

On the other hand, $\left|g\left(x_{1}\right)\right|<15 \leq\left|g^{2}\left(x_{0}\right)\right|$, so we show that $\left|S_{15}\right|=1$. The suffix of length 15 of $g^{2}\left(x_{0}\right)$ is 101101111011011 , which by Corollary 2 is a suffix of $g^{j}\left(x_{1}\right)$ for all $j \geq 2$. Since $15>\left|g\left(x_{1}\right)\right|$, there does not exist an additional member of $S_{15}$ as there did in $S_{10}$. Thus $\left|S_{15}\right|=1$. 


\section{Subword Complexity of $F$}

In this section we determine the subword complexity $c(n)$ of $F$. In section 2 , we defined $F=f^{\omega}(1)$, where $f$ is a morphism such that $f(1)$ begins with 1 . Thus the results of [13] indicate that the order of $c(n)$ can be determined directly from the order of the functions

$$
u(n)=\left|f^{n}(1)\right| \quad \text { and } \quad v(n)=\left|f^{n}(0)\right| .
$$

More precisely, if both $u(n)=\Theta\left(k^{n}\right)$ and $v(n)=\Theta\left(k^{n}\right)$, then $F$ has linear subword complexity. For convenience, denote $u_{n}=u(n)$, and $v_{n}=v(n)$ for all $n \in \mathbb{Z}_{\geq 0}$.

Now $u_{0}=1$, and $u_{1}=\left|1^{s+t-1} 0\right|=s+t$. For $n \geq 2$,

$$
f^{n}(1)=f^{n-1}(f(1))=f^{n-1}\left(1^{s+t-1} 0\right)=f^{n-1}\left(1^{s+t-1}\right) f^{n-2}\left(1^{s}\right),
$$

so $u_{n}=(s+t-1) u_{n-1}+s u_{n-2}=r u_{n-1}+s u_{n-2}$, where $r=s+t-1$. The characteristic polynomial of this recurrence is $x^{2}-r x-s=0$, which has solutions

$$
\alpha=\frac{r+\sqrt{r^{2}+4 s}}{2}, \quad \beta=\frac{r-\sqrt{r^{2}+4 s}}{2} .
$$

Thus for general $n \in \mathbb{Z}_{\geq 0}, u_{n}=c_{1} \alpha^{n}+c_{2} \beta^{n}$, where $c_{1}$ and $c_{2}$ are constants. Solving for $c_{1}$ and $c_{2}$, therefore, we obtain the solutions :

$$
c_{1}=\frac{1}{2}+\frac{r+2}{2 \sqrt{r^{2}+4 s}}, \quad c_{2}=\frac{1}{2}-\frac{r+2}{2 \sqrt{r^{2}+4 s}} .
$$

Now, $r=s+t-1 \geq 1$ implies that $\alpha>1,-1<\beta<0$, and $-\frac{1}{2}<c_{2}<0$. Thus for even $n,-\frac{1}{2}<c_{2} \beta^{n}<0$, and for odd $n, 0<c_{2} \beta^{n}<\frac{1}{2}$. It follows that

$$
u_{n}=c_{1} \alpha^{n}+c_{2} \beta^{n}= \begin{cases}\left\lfloor c_{1} \alpha^{n}\right\rfloor, & n \text { even } \\ \left\lceil c_{1} \alpha^{n}\right\rceil, & n \text { odd } .\end{cases}
$$

Notation. Let $E(x)$ denote the closest integer to $x$.

For every $n \in \mathbb{Z}_{>0}$,

$$
u_{n}=E\left(c_{1} \alpha^{n}\right)
$$

Also, for all $n \geq 1, f^{n}(0)=f^{n-1}(f(0))=f^{n-1}\left(1^{s}\right)$, so $v_{n}=s u_{n-1}$. Thus both $u_{n}=\Theta\left(\alpha^{n}\right)$ and $v_{n}=\Theta\left(\alpha^{n}\right)$, so $F$ has linear subword complexity [13].

We now determine a more precise formula for $c(n)$, using equation (1):

$$
c(n)=2+\sum_{i=1}^{n-1} N(i)=n+1+\sum_{i=1}^{n-1}(N(i)-1) .
$$


Theorem 3 implies that this is equivalent to

$$
c(n)=n+1+k,
$$

where $k$ is the number of integers $m<n$ such that $\left|g^{i}\left(x_{0}\right)\right|<m \leq\left|g^{i}\left(x_{1}\right)\right|$ for some $i \in \mathbb{Z}_{\geq 0}$.

Definition. For every $i \in \mathbb{Z}_{\geq 0}$, let $I_{i}$ denote the interval of integers $m$ satisfying $\left|g^{i}\left(x_{0}\right)\right|<$ $m \leq\left|g^{i}\left(x_{1}\right)\right|$, and let $\left|I_{i}\right|$ denote its length.

Lemma 8. Let $n \in \mathbb{Z}_{>0}$. If $n-1 \leq\left|x_{0}\right|$, or $\left|g^{i}\left(x_{1}\right)\right| \leq n-1 \leq\left|g^{i+1}\left(x_{0}\right)\right|$ for some $i \in \mathbb{Z}_{\geq 0}$, then

$$
c(n)=n+1+(s-1) \sum_{j=0}^{i} u_{j},
$$

where $i$ is the minimal integer such that $\left|g^{i+1}\left(x_{0}\right)\right| \geq n-1$.

Proof. let $k$ be the number of integers $m<n$ such that for some $j \in \mathbb{Z}_{\geq 0}, m \in I_{j}$. Then $k=\sum_{j=0}^{i}\left|I_{j}\right|$. Now, for every $j \in \mathbb{Z}_{\geq 0}$,

$$
\left|I_{j}\right|=\left|g^{j}\left(x_{1}\right)\right|-\left|g^{j}\left(x_{0}\right)\right|
$$

Thus $\left|I_{0}\right|=\left|x_{1}\right|-\left|x_{0}\right|=(s-1) u_{0}$. Similarly, for $j \geq 1$, Lemma 7 (c) implies that

$$
\left|I_{j}\right|=\left|g^{j}\left(x_{1}\right)\right|-\left|g^{j}\left(x_{0}\right)\right|=\left|f^{j}\left(1^{s-1}\right)\right|=(s-1) u_{j} .
$$

Thus $k=\sum_{j=0}^{i}\left|I_{j}\right|=(s-1) \sum_{j=0}^{i} u_{j}$, so our result follows from (4).

Remark. If $s=1$, then $x_{0}=x_{1}$, so for every $m \in \mathbb{Z}_{>0}$, either $m \leq\left|x_{0}\right|$ or $\left|g^{i}\left(x_{1}\right)\right|=$ $\left|g^{i}\left(x_{0}\right)\right| \leq m \leq\left|g^{i+1}\left(x_{0}\right)\right|$ for some $i \in \mathbb{Z}_{\geq 0}$. Thus Lemma 8 implies that in the case $s=1$, $c(n)=n+1$ for all $n \in \mathbb{Z}_{>0}$.

Applying Lemma 8, we analyze $c(n)$ for two different classes of $n$ :

(a) $n=\left|g^{i}\left(x_{0}\right)\right|+1$ for some $i \in \mathbb{Z}_{\geq 0}$, (b) $n=\left|g^{i}\left(x_{1}\right)\right|+1$ for some $i \in \mathbb{Z}_{\geq 0}$. To do so, we define functions $L_{i}(n)$ and $U_{i}(n)$, which are dependent on $i$, and show that $c(n)=L_{i}(n)$ and $c(n)=U_{i}(n)$ in cases (a) and (b) respectively.

Lemma 9. For every $i \in \mathbb{Z}_{\geq 0},\left|g^{i}\left(x_{0}\right)\right|=(s+t-1) \sum_{j=0}^{i} u_{j}$. 
Proof. Lemma 7(b) implies that

$$
\left|g^{i}\left(x_{0}\right)\right|=\sum_{j=0}^{i}\left|f^{j}\left(x_{0}\right)\right|=(s+t-1) \sum_{j=0}^{i}\left|f^{j}(1)\right|=(s+t-1) \sum_{j=0}^{i} u_{j} .
$$

Let $n=\left|g^{i}\left(x_{0}\right)\right|+1$ for some $i \in \mathbb{Z}_{\geq 0}$. Then by Lemmas 8 and 9 , we have

$$
c(n)=\left(1-\frac{(s-1) \sum_{j=0}^{i-1} u_{j}}{(s+t-1) \sum_{j=0}^{i} u_{j}}\right)+\left(1+\frac{(s-1) \sum_{j=0}^{i-1} u_{j}}{(s+t-1) \sum_{j=0}^{i} u_{j}}\right) n .
$$

But (3) implies that for all $m \in \mathbb{Z}_{\geq 0}$,

$$
\sum_{j=0}^{m} u_{j}=\sum_{j=0}^{m} E\left(c_{1} \alpha^{j}\right) \approx c_{1} \frac{\alpha^{m+1}-1}{\alpha-1}
$$

so it follows that

$$
c(n) \approx\left(1-\frac{(s-1)\left(\alpha^{i}-1\right)}{(s+t-1)\left(\alpha^{i+1}-1\right)}\right)+\left(1+\frac{(s-1)\left(\alpha^{i}-1\right)}{(s+t-1)\left(\alpha^{i+1}-1\right)}\right) n .
$$

Definition. For every $i \in \mathbb{Z}_{\geq 0}$,

$$
L_{i}(n)=E\left(\left(1-\frac{(s-1)\left(\alpha^{i}-1\right)}{(s+t-1)\left(\alpha^{i+1}-1\right)}\right)+\left(1+\frac{(s-1)\left(\alpha^{i}-1\right)}{(s+t-1)\left(\alpha^{i+1}-1\right)}\right) n\right) .
$$

Theorem 4. If $n=\left|g^{i}\left(x_{0}\right)\right|+1$ for some $i \in \mathbb{Z}_{\geq 0}$, then $c(n)=L_{i}(n)$.

The proof of Theorem 4 depends on the following two lemmas, which we leave to the reader to verify. Recall that for all $i \in \mathbb{Z}_{\geq 0}, u_{i}=c_{1} \alpha^{i}+c_{2} \beta^{i}$, where $-1<\beta<0$, and $-\frac{1}{2}<c_{2}<0$.

Lemma 10. For every $i \in \mathbb{Z}_{\geq 0}, 0<\sum_{j=0}^{i} \beta^{j} \leq 1$, with equality to 1 if and only if $i=0$.

Lemma 11. $s\left|c_{2}\right|<1 / 2$.

Proof of Theorem 4. If $s=1$, then for all $n, L_{i}(n)=n+1$. But Lemma 8 implies that $c(n)=n+1$ for all $n$, so we are done. To prove the theorem for $s \geq 2$, we first note 
that if $z$ is an integer, then given any real number $x$, proving that $E(x)=z$ is equivalent to proving that $|z-E(x)|<\frac{1}{2}$.

Let $s \geq 2$, and suppose that $n=\left|g^{i}\left(x_{0}\right)\right|+1$ for some $i \in \mathbb{Z}_{\geq 0}$. Then letting $n^{\prime}=$ $n-1=\left|g^{i}\left(x_{0}\right)\right|$, we have $N\left(n^{\prime}\right)=1$ by Theorem 3 , so $c\left(n^{\prime}\right)=c(n)-1$. Thus it suffices to show that

$$
\begin{gathered}
c\left(n^{\prime}\right)=E\left(-\frac{(s-1)\left(\alpha^{i}-1\right)}{(s+t-1)\left(\alpha^{i+1}-1\right)}+\left(1+\frac{(s-1)\left(\alpha^{i}-1\right)}{(s+t-1)\left(\alpha^{i+1}-1\right)}\right) n\right) \\
=n^{\prime}+1+E\left(\frac{(s-1)\left(\alpha^{i}-1\right)}{(s+t-1)\left(\alpha^{i+1}-1\right)} n^{\prime}\right) .
\end{gathered}
$$

Lemma 8 implies that this is equivalent to proving that

$$
(s-1) \sum_{j=0}^{i-1} u_{j}=E\left(\frac{(s-1)\left(\alpha^{i}-1\right)}{(s+t-1)\left(\alpha^{i+1}-1\right)} n^{\prime}\right),
$$

or equivalently,

$$
(s-1)\left|\sum_{j=0}^{i-1} u_{j}-\frac{\left(\alpha^{i}-1\right)}{(s+t-1)\left(\alpha^{i+1}-1\right)} n^{\prime}\right|<\frac{1}{2} .
$$

Now $u_{j}=c_{1} \alpha^{j}+c_{2} \beta^{j}$, and by Lemma $9, n^{\prime}=\left|g^{i}\left(x_{0}\right)\right|=(s+t-1) \sum_{j=0}^{i} u_{j}$. Therefore

$$
(s-1)\left|\sum_{j=0}^{i-1} u_{j}-\frac{\left(\alpha^{i}-1\right)}{(s+t-1)\left(\alpha^{i+1}-1\right)} n^{\prime}\right|=(s-1)\left|c_{2}\left(\sum_{j=0}^{i-1} \beta^{j}-\frac{\alpha^{i}-1}{\alpha^{i+1}-1} \sum_{j=0}^{i} \beta^{j}\right)\right| .
$$

Thus by Lemmas 10 and 11, equation (5) is satisfied.

Let $n=\left|g^{i}\left(x_{1}\right)\right|+1=\left|g^{i}\left(x_{0}\right)\right|+\left|I_{i}\right|+1$ for some $i \in \mathbb{Z}_{\geq 0}$. Lemma 9 implies that $\left|g^{i}\left(x_{0}\right)\right|=(s+t-1) \sum_{j=0}^{i} u_{j}$. Similarly, in the proof of Lemma 8, we saw that $\left|I_{i}\right|=$ $(s-1) u_{i}$. Therefore,

$$
n=(s+t-1) \sum_{j=0}^{i} u_{j}+(s-1) u_{i}+1
$$

Now, Lemma 8 implies that

$$
c(n)=n+1+\frac{(s-1) \sum_{j=0}^{i} u_{j}}{(s+t-1) \sum_{j=0}^{i} u_{j}+(s-1) u_{i}+1} n .
$$

Thus by (3), we have

$$
c(n) \approx n+1+E\left(\frac{c_{1}(s-1) \sum_{j=0}^{i} \alpha^{j}}{c_{1}(s+t-1) \sum_{j=0}^{i} \alpha^{j}+c_{1}(s-1) \alpha^{i}+1} n\right) .
$$


Definition. For every $i \in \mathbb{Z}_{\geq 0}$,

$$
U_{i}(n)=n+1+E\left(\frac{(s-1)\left(\alpha^{i+1}-1\right)}{(s+t-1)\left(\alpha^{i+1}-1\right)+(s-1)\left(\alpha^{i+1}-\alpha^{i}\right)+(\alpha-1) / c_{1}} n\right) .
$$

Theorem 5. If $n=\left|g^{i}\left(x_{1}\right)\right|+1$ for some $i \in \mathbb{Z}_{\geq 0}$, then $c(n)=U_{i}(n)$.

Proof. If $s=1$, then for all $n, U_{i}(n)=n+1$. But Lemma 8 implies that $c(n)=n+1$ for all $n$, so we are done. To prove the theorem for $s \geq 2$, we first note that the two formulas presented in equations $(7)$ and $(8)$ are equivalent.

Now, from Lemma 8 we have $c(n)=n+1+(s-1) \sum_{j=0}^{i} u_{j}$, so it suffices to prove that

$$
(s-1) \sum_{j=0}^{i} u_{j}=E\left(\frac{c_{1}(s-1) \sum_{j=0}^{i} \alpha^{j}}{c_{1}(s+t-1) \sum_{j=0}^{i} \alpha^{j}+c_{1}(s-1) \alpha^{i}+1} n\right),
$$

or equivalently, that

$$
(s-1)\left|\sum_{j=0}^{i} u_{j}-\frac{c_{1} \sum_{j=0}^{i} \alpha^{j}}{c_{1}(s+t-1) \sum_{j=0}^{i} \alpha^{j}+c_{1}(s-1) \alpha^{i}+1} n\right|<\frac{1}{2} .
$$

Since $n=\left|g^{i}\left(x_{1}\right)\right|+1$, equation (6) implies that (9) is equivalent to

$$
(s-1)\left|\sum_{j=0}^{i} u_{j}-\frac{c_{1} \sum_{j=0}^{i} \alpha^{j}\left((s+t-1) \sum_{j=0}^{i} u_{j}+(s-1) u_{i}+1\right)}{c_{1}(s+t-1) \sum_{j=0}^{i} \alpha^{j}+c_{1}(s-1) \alpha^{i}+1}\right|<\frac{1}{2} .
$$

This inequality can be verified by substituting $c_{1} \alpha^{j}+c_{2} \beta^{j}$ for $u_{j}$ and applying Lemmas 10 and 11.

Theorems 4 and 5 imply that for every $i \in \mathbb{Z}_{\geq 0}, c(n)=L_{i}(n)$ and $c(n)=U_{i}(n)$ at, respectively, the beginning and end points of interval $I_{i}$. But Theorem 3 implies that $c(n)$ increases in steps of 2 throughout the entire interval, so it follows that for all $i \in \mathbb{Z}_{\geq 0}$,

$$
\left|g^{i}\left(x_{0}\right)\right|<n \leq\left|g^{i+1}\left(x_{0}\right)\right| \Longrightarrow L_{i}(n) \leq c(n) \leq U_{i}(n) .
$$

Thus denoting

$$
L(n)=\lim _{i \rightarrow \infty} L_{i}(n), \quad \text { and } \quad \mathrm{U}(\mathrm{n})=\lim _{\mathrm{i} \rightarrow \infty} \mathrm{U}_{\mathrm{i}}(\mathrm{n}),
$$

$L(n)$ and $U(n)$ are, respectively, the lower and upper bounds of the asymptotic subword complexity of $F$ as $n$ approaches $\infty$. The precise formulas for these bounds are stated in Theorem 6 , which we leave to the reader to verify. 
Theorem 6. For every $n \in \mathbb{Z}_{>0}$,

$$
\begin{gathered}
L(n)=E\left(\left(1-\frac{s-1}{(s+t-1) \alpha}\right)+\left(1+\frac{s-1}{(s+t-1) \alpha}\right) n\right), \\
U(n)=E\left(\left(1+\frac{(s-1) \alpha}{(2 s+t-2) \alpha-(s-1)}\right) n+1\right) .
\end{gathered}
$$

\section{Conclusion}

For every $s, t \in \mathbb{Z}_{>0}$, the subword complexity $c$ of $F$ is linear in $n$. We presented tight upper and lower bounds for $c(n)$, both for finite $n$, and as an asymptotic value as $n$ approaches infinity. If $s=1$, the lower and upper bounds are equivalent and we have $c(n)=n+1$ for all $n \in \mathbb{Z}_{>0}$. This property follows from the fact that $c(n+1)-c(n)=N(n)=1$ for all $n \in \mathbb{Z}_{>0}$.

If $s \geq 2$, however, the set of positive integers consists of intervals of integers $n$ satisfying $N(n)=1$, alternating with intervals of $n$ over which $N(n)=2$. Thus as $n$ increases, $c(n)$ grows alternately slower and faster, depending on the type of interval in which $n$ is located. In this case, therefore, the lower and upper bounds of $c(n)$ are distinct.

This difference between the cases $s=1$ and $s \geq 2$ is related to a property of the infinite sequences $A$ characterized by $F$. The sequence $A$ is defined to be a Beatty sequence if there exist real $\alpha, \beta$ such that $A_{n}=\lfloor n \alpha+\beta\rfloor$ for all $n \in \mathbb{Z}_{>0}$. It turns out that $A$ is a Beatty sequence if and only if $s=1$, in which case we have $\alpha=\left(2-t+\sqrt{t^{2}+4}\right) / 2$, $\beta=0$ [11]. Since $\sqrt{t^{2}+4}$ is irrational for all $t \in \mathbb{Z}_{>0}$, it follows that $\alpha$ is irrational. In [1] it was shown that every Beatty sequence with irrational $\alpha$ has a characteristic sequence with subword complexity $c(n)=n+1$ for all $n \in \mathbb{Z}_{>0}$. Our result is consistent with this.

The case $s=1$ differs from $s \geq 2$ also in that $A$ is spectral, that is, $\mid\left(A_{k+i}-A_{k}\right)-$ $\left(A_{j+i}-A_{j}\right) \mid \leq 1$ for every $i, j, k \in \mathbb{Z}_{>0}$, if and only if $s=1$ [11]. This is because the set of spectral sequences is precisely the set of Beatty sequences [4]. This motivates the question of whether $A$ has a similar property when $s \geq 2$. Perhaps associated with some of the $A$ sequences for $s \geq 2$ is an integer $m \geq 2$ such that for all $i, j, k,\left|\left(A_{k+i}-A_{k}\right)-\left(A_{j+i}-A_{j}\right)\right| \leq$ $m$.

Another related observation is to estimate, for fixed $m \in \mathbb{Z}_{>0}$ and for every $n \in \mathbb{Z}_{>0}$, the number of increasing sequences of length $n$ such that for all $i, j, k$ with $1 \leq j, k, j+$ $i, k+i \leq n, \quad\left|\left(A_{k+i}-A_{k}\right)-\left(A_{j+i}-A_{j}\right)\right| \leq m$. For $m=1$, the number of such words of length $n$ is Euler's totient function, which is polynomial in $n$ [3], [7]. For $m=2$, however, R. Tijdeman observed (private communication), that the number of such words is exponential in $n$, since the entire set of length- $n$ words with a characteristic sequence beginning with $\{01,10\}^{\lfloor n / 2\rfloor}$ exhibits this property. 


\section{References}

[1] P. Alessandri and V. Berthé, Three distance theorems and combinatorics on words, L'Enseignement Mathématique 44 (1998) 103-132.

[2] J.-P. Allouche, Sur la complexité des suites infinies, Bull. Belg. Math. Soc. 1 (2) (1994) 133-143.

[3] J. Berstel and M. Pocchiola, A geometric proof of the enumeration formula for Sturmian words, Internat. J. Algebra Comput. 3 (1993) 349-355.

[4] M. Boshernitzan and A.S. Fraenkel, A linear algorithm for nonhomogeneous spectra of numbers, J. of Algorithms 5 (1984) 187-198.

[5] H.S.M. Coxeter, The golden section, phyllotaxis and Wythoff's game, Scripta Math. 19 (1953) 135-143.

[6] K. Culik II and A. Salomaa, On infinite words obtained by iterating morphisms, Theoret. Comput. Sci. 19 (1982) 29-38.

[7] A. de Luca and F. Mignosi, Some combinatorial properties of Sturmian words, Theoret. Comput. Sci. 65 (1994) 361-385.

[8] S. Ferenczi, Complexity of sequences and dynamical systems, Discr. Math. 206 (1999) 145-154.

[9] A.S. Fraenkel, How to beat your Wythoff games' opponents on three fronts, Amer. Math. Monthly 89 (1982) 353-361.

[10] A.S. Fraenkel, Systems of numeration, Amer. Math. Monthly 92 (1985) 105114.

[11] A.S. Fraenkel, Heap games, numeration systems and sequences, Annals of Combinatorics 2 (1998) 197-210.

[12] A. Ostrowski, Bemerkungen zur Theorie der diophantischen Approximationen, Abh. Math. Sem. Hamburg 1 (1922) 77-98.

[13] J.-J. Pansiot, Complexité des facteurs des mots infinis engendrés par morphismes itérés, Lecture Notes in Computer Science vol. 172, 1984, pp. 380389.

[14] P. Roth, Every binary pattern of length six is avoidable on the two-letter alphabet, Acta Inform. 29 (1992) 95-107.

[15] W.A. Wythoff, A modification of the game of Nim, Nieuw Arch. Wiskunde 8 (1907) 199-202. 
[16] A.M. Yaglom and I.M. Yaglom, Challenging Mathematical Problems with Elementary Solutions, Vol. II, Holden-Day, San Francisco, translated by J. McCawley, Jr., revised and edited by B. Gordon, 1967. 